\title{
Anthropometric assessment of children aged between 6 and 14 years from a school in Lisbon
}

\author{
Avaliação antropométrica de crianças entre os 6 e 14 anos de idade de um colégio em Lisboa
}

\section{Adriana Costa ${ }^{1}$, Cíntia Ferreira-Pêgo ${ }^{2}$, and Bruno Sousa ${ }^{1,2^{*}}$}

${ }^{1}$ School of Sciences and Health Technologies, Universidade Lusófona de Humanidades e Tecnologias, Av. Campo Grande 376, 1749-024 Lisbon, Portugal; ${ }^{2}$ CBIOS Lusófona's Research Center for Biosciences and Health Technologies, Av. Campo Grande 376, 1749-024 Lisbon, Portugal

* corresponding author: bruno.sousa@ulusofona.pt

\begin{abstract}
As a global epidemic, obesity is currently a significant public health challenge, especially in children. Childhood obesity is related to short term consequences, but also with an increased risk to develop many comorbidities in adult life, such as cardiovascular diseases, type 2 diabetes, strokes, and others. A cross-sectional study to assess the prevalence of normal weight, pre-obesity and obesity in school-aged children was designed. The final sample size analyzed included 81 participants ( 47 boys and 34 girls) aged between 6 and 14 years old. Pre-obesity and obesity were determined according to the BMI cut-off points adopted by the IOTF.
\end{abstract}

Although no significant differences were found, the prevalence of pre-obesity and obesity for the total population studied was $16.00 \%$ and $6.20 \%$, respectively, with $14.60 \%$, and $4.30 \%$ for boys and $17.60 \%$ and $8.80 \%$ for girls. More girls were pre-obese and obese than boys except at ages 9 and 12. The same age that boys presented cardiometabolic risk associated with a WHtR $>0.50$. These results emphasize the importance of nutritional status assessment to develop appropriate actions to prevent childhood obesity.

Keywords: Anthropometry, obesity, pre-obesity, school-aged children, Portugal

\section{Resumo}

A obesidade é atualmente um grande desafio para a saúde pública, principalmente em crianças. A obesidade infantil está relacionada a consequências a curto prazo e a um elevado risco de desenvolver diversas comorbidades na vida adulta, como doenças cardiovasculares, diabetes tipo 2, acidentes vasculares, entre outros. Foi desenvolvido um estudo transversal para avaliar a prevalência de peso adequado, pré-obesidade e obesidade em crianças em idade escolar. O tamanho final da amostra analisada incluiu 81 participantes (47 do sexo masculino e 34 do sexo masculino) com idades entre 6 e 14 anos. Para determinar pré-obesidade e obesidade foram utilizados os pontos de corte internacionais para a idade e sexo da IOTF.

Embora não tenham sido encontradas diferenças significativas entre sexos, a prevalência de pré-obesidade e obesidade foi de $16,00 \%$ e $6,20 \%$, respetivamente para a população total, $14,60 \%$ e $4,30 \%$, respetivamente, para indivíduos do sexo masculino e $17,60 \%$ e $8,80 \%$ respetivamente, para os do sexo feminino. As participantes do sexo feminino apresentavam mais pré-obesidade e obesidade do que os do sexo masculino, exceto aos 9 e 12 anos de idade, e os participantes do sexo masculino de 12 anos apresentaram risco cardiometabólico associado a uma RCE $>0,50$. Esses resultados enfatizam a importância da avaliação do estado nutricional, com a finalidade de desenvolver ações apropriadas para prevenir a obesidade infantil.

Palavras-chave: Antropometria, obesidade, pré-obesidade, crianças, Portugal 


\section{Introduction}

Obesity is an epidemic and thus a major health concern for our society due to its elevated prevalence and multiple health consequences in childhood and adult life $(1,2)$. This is a consequence of inadequate nutrition, in this case over nutrition (3). In 2016, the WHO reported over 340 million children aged 5-19 years worldwide with excess weight (1). Therefore, preventing childhood obesity as well as screening for pre-obesity and obese children at an early phase is of the utmost importance so that strategies and treatments to attain and maintain a healthy weight can be applied (3).

The nutritional status is one of the best health indicators in children and adolescents $(4,5)$. Growth and development are continuous, non-uniform, and largely influenced by food and nutrition (5). Therefore, it is imperative to evaluate every child, whether sick or healthy (6). Easily measured anthropometric measures are an essential element to monitor the health of children as well as to determine their nutritional status (5). Therefore, measurements such as height, weight, waist circumference (WC), and indices like body mass index (BMI) and waist-to-height ratio (WHtR) are essential to assess when studying a population.

Weight and height are excellent indicators of nutritional status as they represent the most important parameter in the definition of pre-obesity and obesity through BMI (5). This anthropometric index, classified for age, is the ratio between weight in kilograms $(\mathrm{kg})$ and height in square meters $\left(\mathrm{m}^{2}\right)$ and, as previously stated, is used to diagnose pre-obesity and obesity. Among other factors, weight and height are deeply influenced by nutrition, especially in periods of rapid growth, so they should be accessed with care. It should be noted that BMI reflects both fat mass and fat-free mass and is not a direct measure of adiposity (5). Lastly, waist circumference is an indicator of central fat and cardiovascular risk, through the WHtR (5).

Taking all this into consideration, the main objective of the present study was to assess the prevalence of normal weight, pre-obesity, and obesity as other anthropometric parameters, from a sample of children aged between 6 and 14 years old from a private school (Real Colégio de Portugal) in Lisbon, Portugal.

\section{Introdução}

A obesidade é uma epidemia e, portanto, uma grande preocupação de saúde para nossa sociedade, devido à sua elevada prevalência e múltiplas consequências na infância e na vida adulta $(1,2)$. Esta patologia é consequência da nutrição inadequada, neste caso da sobrenutrição (3). O último relatório disponível da OMS contabiliza mais de 340 milhões de crianças entre os 5 a 19 anos com excesso de peso, em 2016 (1). Portanto, a prevenção da obesidade infantil e a triagem precoce de crianças com excesso de peso e obesidade são de extrema importância, de modo a que estratégias e tratamentos para atingir e manter um peso saudável possam ser aplicados nestas populações (3).

O estado nutricional é um dos melhores indicadores de saúde em crianças e adolescentes $(4,5)$. O crescimento e o desenvolvimento são contínuos, não uniformes e amplamente influenciados pela alimentação e nutrição (5). Desta forma, é imprescindível avaliar em todas as crianças, saudáveis ou não (6).

As medidas antropométricas de fácil medição são um elemento essencial para monitorizar a saúde das crianças e determinar seu estado nutricional (5). Portanto, medidas como a estatura, peso, perímetro da cintura (PC) e índices como índice de massa corporal (IMC) e razão perímetro da cintura/estatura (RCE) são essenciais de avaliar no estudo de uma população.

Peso e estatura são excelentes indicadores do estado nutricional, pois representam o parâmetro mais importante na definição de pré-obesidade e obesidade através do IMC (5). Esse índice antropométrico, classificado por idade, é a razão entre o peso em quilogramas $(\mathrm{kg})$ e a estatura em metros quadrados $(\mathrm{m})^{2}$ e, como afirmado anteriormente, é usado para diagnosticar pré-obesidade e a obesidade (5). Ainda assim, o peso e a estatura são profundamente influenciados pela nutrição, especialmente em períodos de rápido crescimento, de modo que devem ser avaliados com rigor, o IMC reflete tanto a massa gorda quanto a livre de gordura e, portanto, não é uma medida direta da adiposidade (5). Por fim, o perímetro da cintura é um indicador de gordura central e risco cardiovascular, através da RCE (5).

Tendo em consideração todos estes parâmetros, o principal objetivo do presente estudo foi avaliar a prevalência de peso adequado, pré-obesidade e obesidade, além de outros parâmetros antropométricos, numa amostra de crianças entre 6 e 14 anos de idade do Real Colégio de Portugal. 


\section{Materials and Methods}

\section{Design and study population}

The children's inclusion criteria included written parental consent (following the Declaration of Helsinki) (7), having no apparent illness, and being present in the school on the day of the scheduled visit by the team conducting the measurements. The evaluations took place over two days during the 2019/2020 school year. In the final analysis of the present study, the effective sample size was 81 participants.

\section{Anthropometric parameters assessment}

All the parameters were assessed by a trained nutritionist with support from third-year students pursuing nutrition sciences degrees at the Universidade Lusófona de Humanidades e Tecnologias. Height was assessed using a portable stadiometer (Standard Steel HM01 Ambala, Haryana, India), with values recorded to the nearest millimeter $(0.1 \mathrm{~cm})(8)$. The children were evaluated standing upright, with their backs to the stadiometer and their feet and knees together, their head positioned with the gaze to the horizontal, along with the Frankfurt plane. Weight was measured with a portable electronic scale (seca sensa 804), recording the value to the nearest decigram $(0.1 \mathrm{~kg})(8)$. Waist circumference was measured with the children standing, with the weight distributed by both feet, using a nonextendable measuring tape. The measurement was made at the medium point between the last rib and the iliac crest, in the horizontal plane, after exhalation, and the value was recorded to the nearest millimeter $(0.1$ $\mathrm{cm})(8)$. During all measurements, the children wore light clothing and no footwear (8). The Body Mass Index (BMI) was later calculated using the normalized Quetelet formula weight $(\mathrm{kg}) /$ height $(\mathrm{m})^{2}(8,9)$ from the measurements obtained for each child. Normal weight, pre-obesity, and obesity were identified following the international age and gender cut-off points proposed by the International Obesity Task Force (IOTF) (10). Waist-to-height ratio (WHtR) was also calculated and categorized, considering a cut-off point of 0.5 used for screening pediatric cardiometabolic risk (11).

\section{Statistical analysis}

All data were processed with Microsoft Excel 2011 for Windows (Redmond, WA, USA) and all statistical analyses were made using the IBM SPSS (Statistical Package for Social Sciences, version 23, Chicago, IL, USA) software. The values determined for continuous

\section{Material e Métodos}

\section{Desenho e População em estudo}

Os critérios de inclusão das crianças incluíram o consentimento por escrito dos pais (de acordo com a Declaração de Helsinki) (7), não ter doença aparente e diagnosticada e estar presente no dia marcado da visita à escola pela equipa que conduziu as medições. As avaliações ocorreram durante dois dias do ano letivo de 2019/2020. Na análise final do presente estudo, o tamanho efetivo da amostra foi de 81 participantes.

\section{Avaliação de parâmetros antropométricos}

Todos os parâmetros foram avaliados por um nutricionista treinado com a ajuda de estudantes do $3^{\circ}$ ano da Licenciatura em Ciências da Nutrição da Universidade Lusófona de Humanidades e Tecnologias. A estatura foi avaliada usando um estadiómetro portável (Standard Steel HM01 Ambala, Haryana, India), e o seu valor registado ao milímetro mais próximo $(0,1 \mathrm{~cm})(8)$. As crianças foram avaliadas em pé, de costas para o estadiómetro e com os pés e joelhos juntos, a cabeça posicionada com o olhar na horizontal, ao longo do plano de Frankfurt. O peso foi medido com uma balança eletrónica portátil (seca sensa 804), registando o valor ao decigrama mais próximo $(0,1 \mathrm{~kg})(8)$. O perímetro da cintura foi avaliado com as crianças em pé, com o peso distribuído pelos dois pés, utilizando uma fita métrica não extensível. A medida foi realizada no ponto médio entre a última costela e a crista ilíaca, no plano horizontal, após uma expiração e o valor foi registado ao milímetro mais próximo $(0,1 \mathrm{~cm})(8)$. Durante todas as medições, as crianças tinham roupas leves e não tinham calçado (8).

O Índice de Massa Corporal (IMC) foi posteriormente calculado usando a fórmula Quetelet normalizada de Peso $(\mathrm{kg}) /$ Estatura $(\mathrm{m})^{2}(8,9)$ a partir das medidas obtidas para cada criança. As categorias de peso adequado, pré-obesidade e obesidade foram identificados de acordo com os pontos de corte internacionais para a idade e sexo propostos pela International Obesity Task Force (IOTF) (10). A razão perímetro da cintura/ estatura (RCE) foi também calculada e categorizada considerando um ponto de corte de 0,5 usado no rastreio de risco cardiometabólico pediátrico (11).

\section{Análise estatística}

Todos os dados foram processados no Microsoft Excel 2011 para Windows (Redmond, WA, EUA) 
variables are expressed as means and standard deviation (SD) and for categorical variables as percentages and numbers (n). For continuous variables, the differences in the distribution of the selected characteristics between groups were compared using Student's t-tests or analysis of variance (ANOVA), as appropriate, and Pearson $\chi^{2}$ tests for categorical variables. All statistical tests made were two-tailed and the significance level was set as $\mathrm{p}<0.05$.

\section{Results}

\section{Sample characterization}

A total of 81 children ( 47 boys and 34 girls) from the private school Real Colégio de Portugal with a mean age of 9.70 years (minimum of 6.02 and maximum of 14.08 years) took part in the present study. Children from the $1^{\text {st }}$ cycle (from $1^{\text {st }}$ to $4^{\text {th }}$ grade) were 37 , from the $2^{\text {nd }}$ cycle $\left(5^{\text {th }}\right.$ and $6^{\text {th }}$ grade) 42 and 2 from the $3^{\text {rd }}$ cycle $\left(7^{\text {th }}\right.$ grade). Table 1 summarizes the general characteristics of the studied population according to gender. No statistical differences were found regarding age, height, weight, BMI, WC, or WHtR in the studied population.

\section{Anthropometric measurements}

The anthropometric measurements assessed for each age category, according to gender, are shown in Table 2 . No significant differences were observed in any of the parameters, i.e., height, weight, BMI, WC, WHTR in different age categories. Nevertheless, girls had overall higher average values than boys for every parameter at ages 7, 8, 10, and 11. The opposite was observed at age 9 and age 12, except for height. Also, at age 12 the mean WHtR for boys was 0.51 , the only age/gender above the cut-off point of 0.50 . Additionally, at age 6 , girls had higher average values than boys for every parameter except height and WC.

To simplify the interpretation of the results, the median BMI for each age category can be observed in Figure 1. Girls presented a higher BMI than boys at ages 6, 7, 8,10 , and 11 years old. The opposite was observed at 9 and 12 years old, where median BMI for girls decreases abruptly.

Although non-significant, the prevalence of pre-obesity $(16.00 \%)$ and obesity (6.20\%), categorized by gender and within the entire studied population can be seen in Table 3. Moreover, pre-obesity $(17.60 \%)$ and obesity e todas as análises estatísticas foram realizadas no software IBM SPSS (Statistical Package for the Social Sciences, versão 23, Chicago, IL, EUA). Os valores determinados para variáveis contínuas estão expressos como média e desvio padrão (DP) e para variáveis categóricas como percentagens e valor absoluto (n). Para variáveis contínuas, as diferenças na distribuição das características selecionadas entre os grupos foram comparadas usando os testes t de Student ou a análise de variância (ANOVA), conforme apropriado, e os testes $\chi^{2}$ de Pearson para variáveis categóricas. Todos os testes estatísticos realizados foram testes bicaudais e o nível de significância foi considerado para $\mathrm{p}<0,05$.

\section{Resultados}

\section{Caracterização da amostra}

No total, participaram no estudo 81 crianças (47 do sexo masculino e 34 do sexo feminino) do colégio privado Real Colégio de Portugal, com média de idade de 9,70 anos (idade mínima de 6.02 e máxima de 14.08 anos). As crianças do $1^{\circ}$ ciclo (do $1^{\circ}$ ao $4^{\circ}$ ano) foram 37 , do $2^{\circ}$ ciclo $\left(5^{\circ}\right.$ e $6^{\circ}$ ano $) 42$ e 2 do $3^{\circ}$ ciclo ( $7^{\circ}$ ano). A Tabela 1 resume as características gerais da população estudada de acordo com o sexo. Não foram encontradas quaisquer diferenças estatísticas em relação à idade, estatura, peso, IMC, PC ou RCE na população estudada.

\section{Medidas antropométricas}

As medidas antropométricas avaliadas para cada categoria de idade, de acordo com o sexo, encontramse na Tabela 2. Não foram observadas diferenças significativas em relação às categorias de idade de estatura, peso, IMC, PC, RCE. No entanto, as participantes do sexo feminino apresentaram no geral, valores médios mais altos do que os do sexo masculino para todos os parâmetros para os 7, 8, 10 e 11 anos. $O$ contrário foi observado nos 9 e 12 anos de idade, exceto para a estatura nesta última. Além disso, aos 12 anos, a média do RCE para indivíduos do sexo masculino é de 0,51, o único acima do ponto de corte de 0,50. Adicionalmente, aos 6 anos, as participantes do sexo feminino apresentaram valores médios mais altos do que os do sexo oposto para todos os parâmetros, exceto estatura e PC. Para simplificar a interpretação dos resultados, o IMC médio para cada categoria de idade é mostrado na Figura 1.

As participantes do sexo feminino apresentaram IMC maior que os participantes do sexo masculino para os 6 , 
Table 1 - General characteristics of the study population according to gender

Tabela 1 - Características gerais da população estudada de acordo com o sexo.

\begin{tabular}{lcccc}
\hline & $\begin{array}{c}\text { All population / } \\
\text { População total } \\
(\mathrm{n}=81)\end{array}$ & $\begin{array}{c}\text { Boys / } \\
\text { Rapazes } \\
(\mathrm{n}=47)\end{array}$ & $\begin{array}{c}\text { Girls / } \\
\text { Raparigas } \\
(\mathrm{n}=34)\end{array}$ & p-valuea \\
\hline Age / Idade, years / anos & $9.70(2.15)$ & $9.58(2.14)$ & $9.86(2.19)$ & 0.567 \\
Height / Estatura, m & $1.39(0.13)$ & $1.38(0.13)$ & $1.41(0.14)$ & 0.241 \\
Weight / Peso, kg & $36.60(12.82)$ & $35.34(13.31)$ & $38.34(12.10)$ & 0.301 \\
BMI / IMC, kg /m & $18.35(3.82)$ & $18.05(4.16)$ & $18.7(3.31)$ & 0.424 \\
WC / PC, cm & $64.17(10.19)$ & $63.97(11.05)$ & $64.44(9.03)$ & 0.838 \\
WHtR / RCE & $0.461(0.05)$ & $0.463(0.05)$ & $0.46(0.05)$ & 0.612 \\
Age categories / & & & & \\
Categorias de idade, \% (n) & & & & \\
6 years / anos & $16.00(13)$ & $17.00(8)$ & $14.70(5)$ & \\
7 years / anos & $11.10(9)$ & $10.60(5)$ & $11.80(4)$ & \\
8 years / anos & $11.10(9)$ & $10.60(5)$ & $11.80(4)$ & \\
9 years / anos & $8.60(7)$ & $12.80(6)$ & $2.90(1)$ & \\
10 years / anos & $16.00(13)$ & $14.90(7)$ & $17.60(6)$ & \\
11 years / anos & $25.90(21)$ & $23.40(11)$ & $29.40(10)$ & \\
12 years / anos & $8.60(7)$ & $8.50(4)$ & $8.80(3)$ & \\
13 years / anos & $1.20(1)$ & $0.00(0)$ & $2.90(1)$ & \\
14 years / anos & $1.20(1)$ & $2.21(1)$ & $0.00(0)$ & \\
Education Level / Nível de & & & & \\
Escolaridade, \% (n) & & & & \\
1st Cycle / 10 Ciclo & $45.70(37)$ & $48.90(23)$ & $41.20(14)$ & \\
2nd Cycle / 2o Ciclo & $51.90(42)$ & $48.90(23)$ & $55.90(19)$ & \\
3rd Cycle / 3o Ciclo & $2.50(2)$ & $2.10(1)$ & $2.90(2)$ & \\
\hline
\end{tabular}

Data expressed as means (SD) or percentages (n). Abbreviations: BMI, body mass index; WC, waist circumference and WHtR, waist-to-height ratio. ${ }^{a} \mathrm{p}$-values for comparisons between groups were tested by Student's t-test or Pearson $\chi^{2}$ as appropriate. /

Dados expressos em média (DP) ou percentagens (n). Abreviaturas: IMC, Índice de Massa Corporal; PC, Perímetro da cintura e RCE, Razão

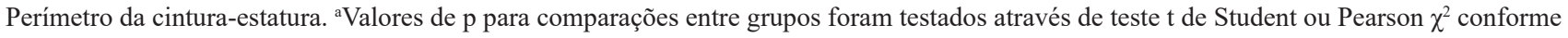
apropriado.

$(8.80 \%)$ were more prevalent in girls than in boys ( $14.90 \%$ and $4.30 \%$, respectively) but without statistical significance. These results can also be visualized in Figure 2 .

In the same way, a statistical analysis to determine the prevalence of cardiometabolic risk by gender was made. The prevalence of cardiometabolic risk associated with a WHtR above 0.50 was $18.50 \%$ (14.90\% for boys and $23.50 \%$ for girls), however, these results were nonsignificant $(\mathrm{p}=0.323)$ (Data not shown).

When the analysis taking into consideration overweight (pre-obesity and/or obesity) in the same category versus normal-weight individuals was performed, total population (not subdivided by age categories) still demonstrated that girls $(26.50 \%)$ were overall
7, 8, 10 e 11 anos. O contrário foi observado nos 9 e 12 anos, onde as medias do IMC das participantes do sexo feminino diminuem abruptamente.

Embora não estatisticamente significativa, a prevalência de pré-obesidade $(16,00 \%)$ e obesidade $(6,20 \%)$, categorizadas por género e para toda a população estudada, pode ser vista na Tabela 3. Além disso, a préobesidade e a obesidade foram mais prevalentes no sexo feminino $(17,60 \%$ e $8,80 \%$, respetivamente) do que no sexo masculino ( $14,90 \%$ e $4,30 \%$, respetivamente), mas sem significância estatística. Esses resultados de forma gráfica podem ser visualizados na Figura 2.

Da mesma forma, foi feita uma análise estatística para determinar a prevalência de risco cardiometabólico para cada sexo. A prevalência de risco cardiometabólico 
Table 2 - Anthropometric measurements according to gender and age categories.

Tabela 2- Dados antropométricos de acordo com o sexo e categorias de idade.

\begin{tabular}{|c|c|c|c|c|}
\hline & $\begin{array}{l}\text { All population / } \\
\text { População total }\end{array}$ & $\begin{array}{c}\text { Boys / } \\
\text { Rapazes }\end{array}$ & $\begin{array}{c}\text { Girls / } \\
\text { Raparigas }\end{array}$ & $p$-value ${ }^{a}$ \\
\hline 6 years / anos & $(n=13)$ & $(n=8)$ & $(n=5)$ & \\
\hline Height / Estatura & $1.20(0.05)$ & $1.21(0.06)$ & $1.19(0.03)$ & 0.626 \\
\hline Weight / Peso & $22.82(2.37)$ & $22.30(2.80)$ & $23.64(1.34)$ & 0.344 \\
\hline $\mathrm{BMI} / \mathrm{IMC}$ & $15.87(1.38)$ & $15.36(1.57)$ & $16.68(0.25)$ & 0.094 \\
\hline WC / PC & $55.62(3.05)$ & $55.75(2.62)$ & $55.40(3.97)$ & 0.850 \\
\hline WHtR / RCE & $0.46(0.02)$ & $0.46(0.03)$ & $0.47(0.02)$ & 0.891 \\
\hline 7 years / anos & $(n=9)$ & $(n=5)$ & $(n=4)$ & \\
\hline Height / Estatura & $1.26(0.04)$ & $1.26(0.04)$ & $1.28(0.04)$ & 0.475 \\
\hline Weight / Peso & $25.23(2.91)$ & $23.96(2.37)$ & $26.83(3.00)$ & 0.153 \\
\hline $\mathrm{BMI} / \mathrm{IMC}$ & $15.75(1.05)$ & $15.18(0.56)$ & $16.46(1.13)$ & 0.060 \\
\hline$W C / P C$ & $57.33(2.83)$ & $56.40(2.07)$ & $58.50(3.51)$ & 0.298 \\
\hline WHtR / RCE & $0.45(0.02)$ & $0.45(0.01)$ & $0.46(0.02)$ & 0.477 \\
\hline 8 years / anos & $(n=9)$ & $(n=5)$ & $(n=4)$ & \\
\hline Height / Estatura & $1.33(0.06)$ & $1.32(0.07)$ & $1.35(0.05)$ & 0.505 \\
\hline Weight / Peso & $31.79(6.12)$ & $30.46(6.85)$ & $33.45(5.53)$ & 0.503 \\
\hline BMI / IMC & $17.8(2.27)$ & $17.33(2.69)$ & $18.32(1.84)$ & 0.551 \\
\hline$W C / P C$ & $62.06(6.27)$ & $60.40(7.23)$ & $64.13(4.97)$ & 0.412 \\
\hline WHtR / RCE & $0.47(0.04)$ & $0.46(0.04)$ & $0.48(0.03)$ & 0.469 \\
\hline 9 years / anos & $(n=7)$ & $(n=6)$ & $(n=1)$ & \\
\hline Height / Estatura & $1.38(0.03)$ & $1.39(0.03)$ & $1.36(0.00)$ & 0.459 \\
\hline Weight / Peso & $35.04(5.93)$ & $36.05(5.81)$ & $29.00(0.00)$ & 0.312 \\
\hline $\mathrm{BMI} / \mathrm{IMC}$ & $18.30(2.91)$ & $18.73(2.92)$ & $15.68(0.00)$ & 0.378 \\
\hline$W C / P C$ & $63.71(5.07)$ & $64.33(5.26)$ & $60.00(0.00)$ & 0.480 \\
\hline WHtR / RCE & $0.46(0.03)$ & $0.46(0.03)$ & $0.44(0.00)$ & 0.572 \\
\hline 10 years / anos & $(n=13)$ & $(n=7)$ & $(n=6)$ & \\
\hline Height / Estatura & $1.44(0.08)$ & $1.41(0.06)$ & $1.47(0.09)$ & 0.199 \\
\hline Weight / Peso & $40.71(11.53)$ & $35.61(9.57)$ & $46.65(11.43)$ & 0.084 \\
\hline $\mathrm{BMI} / \mathrm{IMC}$ & $19.38(4.16)$ & $17.60(3.19)$ & $21.46(4.44)$ & 0.097 \\
\hline$W C / P C$ & $67.23(10.51)$ & $64.79(11.75)$ & $70.08(9.01)$ & 0.388 \\
\hline WHtR / RCE & $0.47(0.07)$ & $0.46(0.06)$ & $0.47(0.07)$ & 0.565 \\
\hline 11 years / anos & $(n=21)$ & $(n=11)$ & $(n=10)$ & \\
\hline Height / Estatura & $1.50(0.07)$ & $1.48(0.07)$ & $1.52(0.06)$ & 0.199 \\
\hline Weight / Peso & $44.54(9.66)$ & $42.36(9.10)$ & $46.94(10.15)$ & 0.290 \\
\hline BMI / IMC & $19.62(3.01)$ & $19.11(2.66)$ & $20.19(3.40)$ & 0.428 \\
\hline$W C / P C$ & $68.67(9.88)$ & $67.91(9.54)$ & $69.50(10.69)$ & 0.722 \\
\hline WHtR / RCE & $0.46(0.06)$ & $0.46(0.05)$ & $0.46(0.07)$ & 0.988 \\
\hline 12 years / anos & $(n=7)$ & $(n=4)$ & $(n=3)$ & \\
\hline Height / Estatura & $1.54(0.06)$ & $1.53(0.09)$ & $1.56(0.03)$ & 0.622 \\
\hline Weight / Peso & $50.60(18.54)$ & $57.13(22.63)$ & $41.90(8.02)$ & 0.324 \\
\hline $\mathrm{BMI} / \mathrm{IMC}$ & $21.24(8.16)$ & $24.27(9.94)$ & $17.18(2.93)$ & 0.294 \\
\hline$W C / P C$ & $71.57(17.45)$ & $78.00(21.73)$ & $63.00(3.61)$ & 0.300 \\
\hline WHtR / RCE & $0.46(0.11)$ & $0.51(0.13)$ & $0.40(0.02)$ & 0.252 \\
\hline 13 years / anos & $(n=1)$ & $(n=0)$ & $(n=1)$ & \\
\hline Height / Estatura & $1.54(0.00)$ & - & $1.54(0.00)$ & b \\
\hline Weight / Peso & $40.40(0.00)$ & - & $40.40(0.00)$ & b \\
\hline $\mathrm{BMI} / \mathrm{IMC}$ & $17.03(0.00)$ & - & $17.03(0.00)$ & b \\
\hline$W C / P C$ & $59.00(0.00)$ & - & $59.00(0.00)$ & $b$ \\
\hline WHtR / RCE & $0.38(0.00)$ & - & $0.38(0.00)$ & $\mathrm{b}$ \\
\hline 14 years / anos & $(n=1)$ & $(n=1)$ & $(n=0)$ & \\
\hline Height / Estatura & $1.58(0.00)$ & $1.58(0.00)$ & - & $\mathrm{b}$ \\
\hline Weight / Peso & $50.30(0.00)$ & $50.30(0.00)$ & - & $b$ \\
\hline $\mathrm{BMI} / \mathrm{IMC}$ & $20.15(0.00)$ & $20.15(0.00)$ & - & $b$ \\
\hline$W C / P C$ & $78.00(0.00)$ & $78.00(0.00)$ & - & $\mathrm{b}$ \\
\hline WHtR / RCE & $0.49(0.00)$ & $0.49(0.00)$ & - & $b$ \\
\hline
\end{tabular}

Data expressed as means (SD). Height expressed in meters (m), weight in kilograms ( $\mathrm{kg}$ ), BMI in $\mathrm{kg} / \mathrm{m}^{2}, \mathrm{WC}$ in centimeters ( $\mathrm{cm}$ ) and WHtR is a ratio. Abbreviations: BMI, body mass index; WC, waist circumference and WHtR, waist-to- height ratio. ${ }^{a} \mathrm{p}$-values for comparisons between groups were tested by Student's t-test. /

Dados expressos como média (DP). Estatura expressa em metros (m), peso em quilogramas ( $\mathrm{kg}$ ), IMC em $\mathrm{kg} / \mathrm{m}^{2}$, PC em centímetros $(\mathrm{cm})$ e RCE é uma razão. Abreviaturas: IMC, Índice de Massa Corporal; PC, Perímetro da cintura e RCE, Razão Perímetro da CinturalEstatura. ${ }^{\mathrm{a}}$ valores-p para comparações entre grupos foram testados através do teste $t$ de Student. 


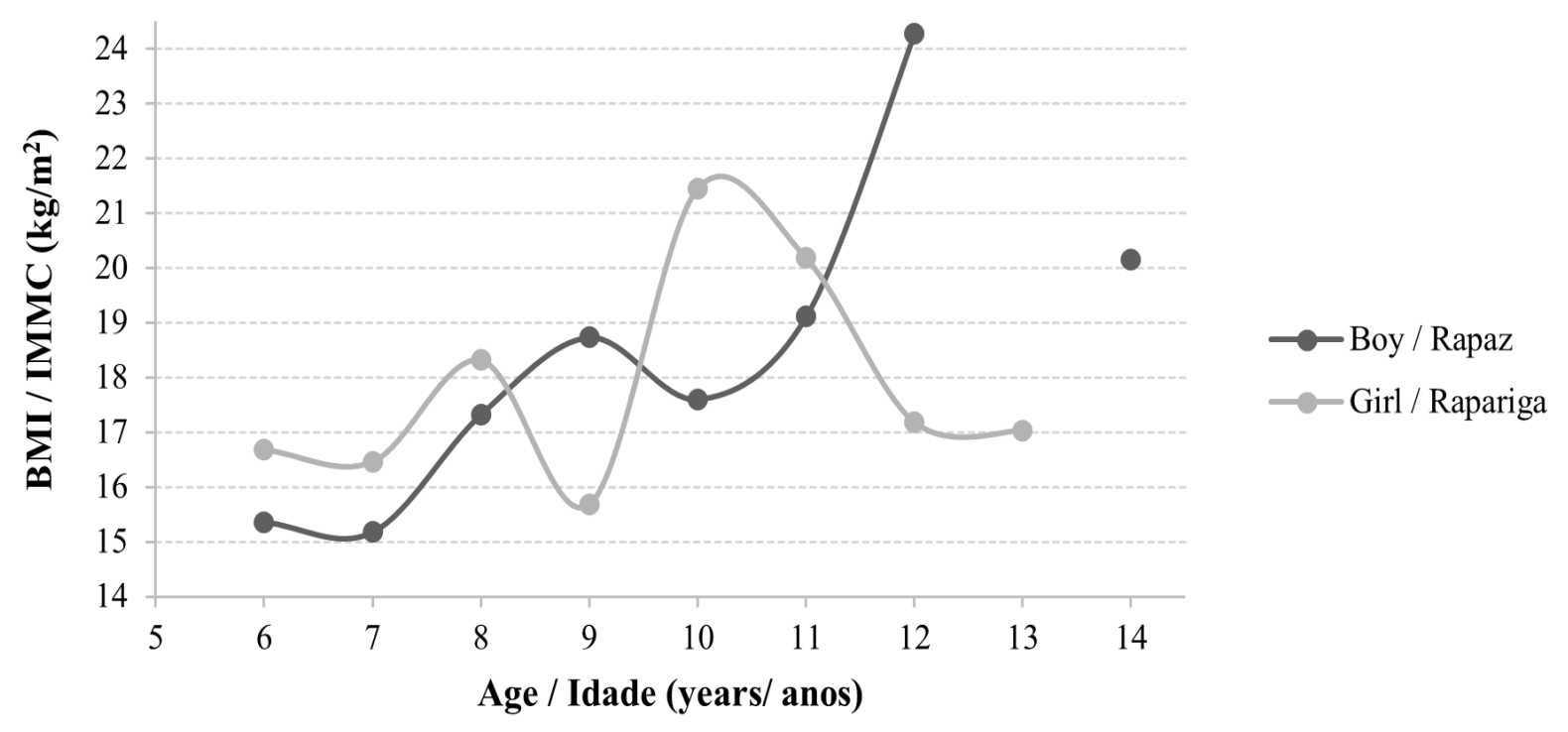

Figure 1 - Median BMI values for each age category by gender.

Figura 1 - Valores médios de IMC para cada categoria de idade por sexo.

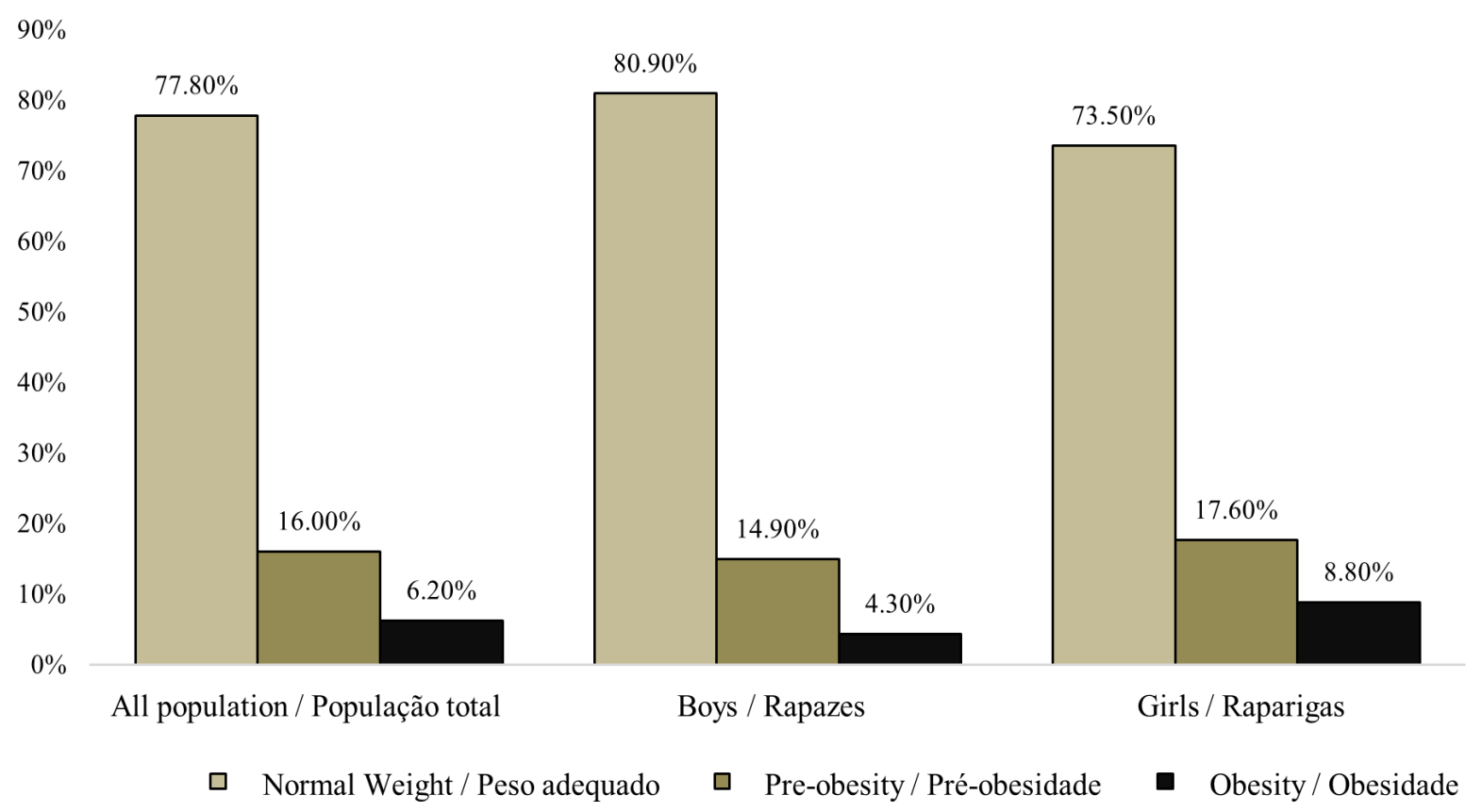

Figure 2 - Percentage of individuals with normal weight, overweight and obesity, according to IOTF criteria (10) categorized by gender.

Figura 2 - Percentagem de indivíduos com peso adequado, pré-obesidade ou obesidade de acordo com os critérios da IOTF categorizada por sexo (10). 
Table 3 - Prevalence of normal weight, overweight and obesity ${ }^{\mathrm{b}}$ according to gender and age categories. Tabela 3 - Prevalência de peso adequado, pré-obesidade e obesidade ${ }^{b}$ de acordo com o sexo e categorias de idade.

\begin{tabular}{|c|c|c|c|c|}
\hline & $\begin{array}{l}\text { All population / } \\
\text { População total }\end{array}$ & $\begin{array}{c}\text { Boys / } \\
\text { Rapazes }\end{array}$ & $\begin{array}{c}\text { Girls / } \\
\text { Raparigas }\end{array}$ & $\begin{array}{c}\mathrm{p} \text {-value / } \\
\text { valores- } \mathrm{p}^{\mathrm{a}} \\
\end{array}$ \\
\hline \multicolumn{5}{|l|}{6 years / anos } \\
\hline Normal Weight / Peso adequado & $100.00(13)$ & $100.00(8)$ & $100.00(5)$ & \\
\hline Overweight / Pré-obesidade & $0.00(0)$ & $0.00(0)$ & $0.00(0)$ & - \\
\hline Obesity / Obesidade & $0.00(0)$ & $0.00(0)$ & $0.00(0)$ & \\
\hline \multicolumn{5}{|l|}{7 years / anos } \\
\hline Normal Weight / Peso adequado & $88.90(8)$ & $100.00(5)$ & $75.00(3)$ & \\
\hline Overweight / Pré-obesidade & $11.10(1)$ & $0.00(0)$ & $25.00(1)$ & 0.236 \\
\hline Obesity / Obesidade & $0.00(0)$ & $0.00(0)$ & $0.00(0)$ & \\
\hline \multicolumn{5}{|l|}{8 years / anos } \\
\hline Normal Weight / Peso adequado & $66.70(6)$ & $60.00(3)$ & $75.00(3)$ & \\
\hline Overweight / Pré-obesidade & $33.30(3)$ & $40.00(2)$ & $25.00(1)$ & 0.635 \\
\hline Obesity / Obesidade & $0.00(0)$ & $0.00(0)$ & $0.00(0)$ & \\
\hline \multicolumn{5}{|l|}{9 years / anos } \\
\hline Normal Weight / Peso adequado & $57.10(4)$ & $50.00(3)$ & $100.00(1)$ & \\
\hline Overweight / Pré-obesidade & $42.90(3)$ & $50.00(3)$ & $0.00(0)$ & 0.350 \\
\hline Obesity / Obesidade & $0.00(0)$ & $0.00(0)$ & $0.00(0)$ & \\
\hline \multicolumn{5}{|l|}{10 years / anos } \\
\hline Normal Weight / Peso adequado & $69.20(9)$ & $85.70(6)$ & $50.00(3)$ & \\
\hline Overweight / Pré-obesidade & $15.40(2)$ & $14.30(1)$ & $16.70(1)$ & 0.230 \\
\hline Obesity / Obesidade & $15.40(2)$ & $0.00(0)$ & $33.30(2)$ & \\
\hline \multicolumn{5}{|l|}{11 years / anos } \\
\hline Normal Weight / Peso adequado & $76.20(16)$ & $90.90(10)$ & $60.00(6)$ & \\
\hline Overweight / Pré-obesidade & $19.00(4)$ & $9.10(1)$ & $30.00(3)$ & 0.228 \\
\hline Obesity / Obesidade & $4.80(1)$ & $0.00(0)$ & $10.00(1)$ & \\
\hline \multicolumn{5}{|l|}{12 years / anos } \\
\hline Normal Weight / Peso adequado & $71.40(5)$ & $50.00(2)$ & $100.00(3)$ & \\
\hline Overweight / Pré-obesidade & $0.00(0)$ & $0.00(0)$ & $0.00(0)$ & 0.147 \\
\hline Obesity / Obesidade & $28.60(2)$ & $50.00(2)$ & $0.00(0)$ & \\
\hline \multicolumn{5}{|l|}{13 years / anos } \\
\hline Normal Weight / Peso adequado & $100.00(1)$ & $0.00(0)$ & $100.00(1)$ & \\
\hline Overweight / Pré-obesidade & $0.00(0)$ & $0.00(0)$ & $0.00(0)$ & - \\
\hline Obesity / Obesidade & $0.00(0)$ & $0.00(0)$ & $0.00(0)$ & \\
\hline \multicolumn{5}{|l|}{14 years / anos } \\
\hline Normal Weight / Peso adequado & $100.00(1)$ & $100.00(1)$ & $0.00(0)$ & \\
\hline Overweight / Pré-obesidade & $0.00(0)$ & $0.00(0)$ & $0.00(0)$ & - \\
\hline Obesity / Obesidade & $0.00(0)$ & $0.00(0)$ & $0.00(0)$ & \\
\hline \multicolumn{5}{|l|}{ TOTAL } \\
\hline Normal Weight / Peso adequado & $77.80(63)$ & $80.90(38)$ & $73.50(25)$ & \\
\hline Overweight / Pré-obesidade & $16.00(13)$ & $14.90(7)$ & $17.60(6)$ & 0.639 \\
\hline Obesity / Obesidade & $6.20(5)$ & $4.30(2)$ & $8.80(3)$ & \\
\hline
\end{tabular}

Data expressed as percentages (n). ${ }^{\mathrm{a}} \mathrm{p}$-values for comparisons between groups were tested by Pearson $\chi^{2}$. ${ }^{\mathrm{b}}$ Normal weight, overweight and obesity were categorized according to IOTF criteria (10). ${ }^{b} \mathrm{p}$-value impossible to compute due fewer than two groups for dependent variables. /

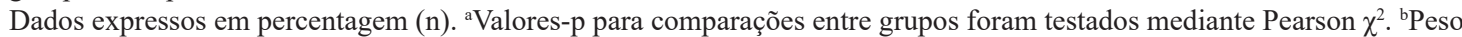

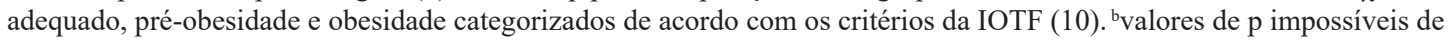
calcular pois existem menos de dois grupos para as variáveis dependentes. 
heavier than boys (19.10\%) although not significantly $(\mathrm{p}=0.434)$ (Data not shown).

Table 4 shows the prevalence of pre-obesity and obesity and cardiometabolic risk associated with WHtR, categorized by education level. The children from the $2^{\text {nd }}$ Cycle have the highest prevalence of pre-obesity and obesity and cardiometabolic risk. None of the measures presented significant differences.

\section{Discussion}

Girls were more pre-obese and obese, and these results were not related to age. These results are consistent with previously published studies, showing a higher prevalence of pre-obesity and obesity in early and middle childhood girls $(12,13)$. This conclusion does not apply at the age of 9 and 12 years, likely due to the reduced sample, creating large disparities between genders and weight clusters (normal weight, preobesity, and obesity) in these age categories.

Regarding age relation, previous studies in Portugal described statistically higher BMI values in girls at 4 and 7 years old, whereas at 10 years old boys were heavier than girls (14). This suggests a possible relation between pre-obesity and age that was not observed in this work. associado a um RCE acima de 0,50 foi de $18,50 \%$ (14,90\% para o sexo masculino e $23,50 \%$ para o sexo feminino), contudo as diferenças entre grupos não foram significativas $(p=0,323)$ (dados não apresentados).

Quando a análise considerou o excesso de peso (pré-obesidade + obesidade) versus indivíduos com peso adequado foi realizada, a população total (não subdividida por categorias de idade) continuou a demonstrar que as participantes do sexo feminino $(26,50 \%)$ apresentaram maior peso corporal do que os participantes do sexo masculino $(19,10 \%)$ embora não tenham sido observadas diferenças estatisticamente significativas $(p=0,434)$ (dados não apresentados).

A Tabela 4 mostra a prevalência de excesso de peso e obesidade e de risco cardiometabólico associado à RCE, categorizados por nível de escolaridade. As crianças do $2^{\circ}$ Ciclo apresentam a maior prevalência de excesso de peso e obesidade e de risco cardiometabólico. Nenhuma das medidas apresentou diferenças significativas.

\section{Discussão}

As participantes do sexo feminino apresentaram mais excesso de peso (pré-obesidade e obesidade), independentemente da idade. Esses resultados são consistentes com estudos anteriores, segundo os quais

Table 4 - Prevalence of normal weight, overweight, obesity ${ }^{\mathrm{b}}$ and cardiometabolic risk ${ }^{\mathrm{c}}$ according to $^{-}$ education level.

Tabela 4 - Prevalência de peso adequado, pré-obesidade, obesidade ${ }^{b}$ e risco cardiometabólico ${ }^{c}$ de acordo com o nível de escolaridade.

\begin{tabular}{|c|c|c|c|c|c|}
\hline & \multicolumn{5}{|c|}{ Education Level / Nível de Escolaridade } \\
\hline & $\begin{array}{l}\text { All population / } \\
\text { População total }\end{array}$ & $\begin{array}{c}1^{\text {st }} \text { Cycle / } \\
10 \text { Ciclo }\end{array}$ & $\begin{array}{c}2^{\text {nd }} \text { Cycle / } \\
20 \text { Ciclo }\end{array}$ & $\begin{array}{c}3^{\text {rd }} \text { Cycle / } \\
\text { 3o Ciclo }\end{array}$ & p-value \\
\hline \multicolumn{6}{|l|}{$\mathrm{BMI} / \mathrm{IMC}$} \\
\hline $\begin{array}{l}\text { Normal Weight / } \\
\text { Peso adequado }\end{array}$ & $77.80(63)$ & $83.80(31)$ & $71.40(30)$ & $100.00(2)$ & \multirow{3}{*}{0.241} \\
\hline Overweight / Pré-obesidade & $16.00(13)$ & $16.20(6)$ & 16.70 (7) & $0.00(0)$ & \\
\hline Obesity / Obesidade & $6.50(5)$ & $0.00(0)$ & $11.90(5)$ & $0.00(0)$ & \\
\hline \multicolumn{6}{|l|}{ WHtR / RCE } \\
\hline $\begin{array}{l}\text { Without cardiometabolic risk / } \\
\text { Sem risco cardiometabólico }\end{array}$ & $81.50(66)$ & $91.90(34)$ & $71.40(30)$ & $100.00(2)$ & \multirow{2}{*}{0.052} \\
\hline $\begin{array}{l}\text { With cardiometabolic risk / } \\
\text { Com risco cardiometabólico }\end{array}$ & $18.50(15)$ & $8.10(3)$ & $28.60(12)$ & $0.00(0)$ & \\
\hline
\end{tabular}

Data expressed as percentages (n). ${ }^{\mathrm{a} p}$-values for comparisons between groups tested by Pearson $\chi^{2}$. ${ }^{\mathrm{b} N o r m a l}$ weight, overweight and obesity categorized according to IOTF criteria (10). ${ }^{\mathrm{C} C}$ ardiometabolic risk categorized according to the cut-off point of 0.5 (11) / Dados expressos em percentagem (n). ${ }^{a}$ Valores de p para comparações entre grupos testados através de Pearson $\chi^{2}$. ${ }^{b}$ Peso adequado,

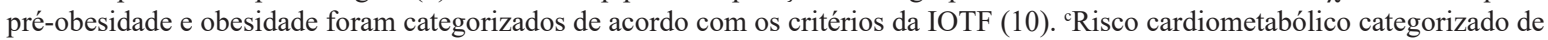
acordo com o ponto de corte de 0.5 (11). 
Boys with 12 years presented a slight cardiometabolic risk, which is associated with childhood obesity and all its short term comorbidities, such as high cholesterol and blood pressure (15). Moreover, obese children are more likely to become obese adults, which increases the risk of many health problems, including cardiovascular disease, type 2 diabetes, and stroke (11).

Regarding the overall lower prevalence of pre-obesity and/ or obesity found in this sample, these results are likely associated to several sociodemographic variables such as economic status and educational level, and other behaviors related to eating patterns, sedentary lifestyle, physical activity, and even sleeping habits, as found in previous studies (16). However, no data regarding these aspects were collected in the present study. Therefore, it is not certain that those aspects explain these results. Further studies are needed to better clarify the sociodemographic variables responsible for these differences in this population.

Furthermore, older children, particularly those in the $2^{\text {nd }}$ cycle, were more prone to pre-obesity and obesity and presented more cardiometabolic risk compared with younger children ( $1^{\text {st }}$ cycle). These results may be due to the higher susceptibility of younger children (6 to 9 years) to parental dietary barriers and strategies to promote healthy eating, whereas older children (10 to 12 years), i.e., those in pre-adolescence, are more likely to go against these efforts and ideals imposed by parents, thus compromising adequate weight (17). This indicates that pre-adolescence represents a window of opportunity to strengthen obesity prevention strategies.

To the best of our knowledge, the present study is the first to assess exclusively anthropometric data and to describe the relationship between education level and the prevalence of pre-obesity and obesity in school children (aged between 6 and 14 years old) from this private institution. Nevertheless, future studies with larger samples should be considered to obtain more conclusive and significant results. as participantes do sexo feminino apresentam maior prevalência de excesso de peso no início e no meio da infância $(12,13)$. Essa conclusão não se aplica aos 9 e 12 anos de idade, provavelmente devido à reduzida amostra que criou grandes disparidades entre géneros e classes de peso nessas categorias de idade.

Em relação à idade, estudos anteriores em Portugal descreveram valores estatisticamente mais altos de IMC em indivíduos do sexo feminino de 4 e 7 anos do que em indivíduos do sexo masculino, enquanto aos 10 anos os participantes do sexo masculino tinham mais peso do que as participantes do sexo feminino (14). Isso sugere uma possível relação entre excesso de peso e idade, a qual não foi observada neste trabalho.

Participantes do sexo masculino com 12 anos apresentaram risco cardiometabólico, o qual está associado à obesidade infantil e a todas as suas comorbidades a curto prazo, como colesterol e pressão arterial elevados (15). Além disso, crianças obesas têm maior probabilidade de se tornarem adultos obesos, o que aumenta o risco de muitos problemas de saúde nessa idade, incluindo doenças cardiovasculares, diabetes tipo 2 e acidente vascular (11).

Em relação à baixa prevalência geral de excesso de peso e/ ou obesidade encontrada nesta amostra, esses resultados provavelmente estão associados a várias variáveis sociodemográficas, como o nível económico e o nível de escolaridade e ainda outros comportamentos relacionados com padrões alimentares, estilo de vida sedentário, atividade física e até hábitos de sono, como reportado em estudos anteriores (16). No entanto, nenhum dado sobre estes aspetos foi recolhido no presente estudo. Portanto, não é certo que esses aspetos expliquem estes resultados. Isto reflete a necessidade de novos estudos, para melhor esclarecer as variáveis sociodemográficas responsáveis por essas diferenças nessa população.

Além disso, as crianças mais velhas, principalmente as do $2^{\circ}$ ciclo, eram mais propensas a ter excesso de peso e apresentavam maior risco cardiometabólico em comparação com as crianças mais jovens ( $1^{\circ}$ ciclo). Esses resultados podem ser explicados devido à maior suscetibilidade que as crianças mais jovens ( 6 a 9 anos) têm às barreiras e estratégias dos pais para promover uma alimentação saudável, enquanto as crianças mais velhas (10 a 12 anos), ou seja, na pré-adolescência, tendem a ir contra esses esforços e ideais impostos pelos pais, assim como uma maior independência financeira, comprometendo desta forma o peso adequado (17). Isso indica que a pré-adolescência representa uma 


\section{Conclusion}

The results of this study reveal a high prevalence of pre-obesity and obesity among girls, especially those in pre-adolescence. These results suggest a need to develop interventions to successfully control childhood obesity, adapted to the characteristics of this population.

\section{Authors Contributions Statement}

B.S, conceptualization and study design; B.S, A.C, experimental implementation; C.F-P data analysis; A.C, C.F-P drafting, editing, and reviewing; A.C, C.F-P figures and graphics; C.F-P supervision and final writing.

\section{Funding}

Not applicable.

\section{Acknowledgments}

The authors acknowledge all the participants and collaborators involved in the study, especially the students from the 3rd year of the Nutrition Sciences degree of the Universidade Lusófona de Humanidades e Tecnologias.

\section{Conflict of Interests}

A.C., C.F.-P., and B.S. report no conflicts of interest. janela de oportunidade para fortalecer estratégias de prevenção da obesidade. Até onde sabemos, o presente estudo é o primeiro a avaliar exclusivamente dados antropométricos e a descrever a relação entre o nível de escolaridade e a prevalência de excesso de peso em crianças em idade escolar (6 a 14 anos) desta instituição privada. Como os resultados desta análise foram limitados pela amostra reduzida e não representativa, novos estudos com maiores amostras devem ser considerados para obter resultados mais conclusivos e significativos.

\section{Conclusões}

Os resultados deste estudo revelam uma alta prevalência de pré-obesidade e obesidade entre participantes do sexo feminino, principalmente na pré-adolescência. Estes resultados, alertam para a necessidade de desenvolver intervenções para controlar eficazmente a obesidade infantil, adaptada às características desta população.

\section{Declaração sobre as contribuições do autor}

B.S, conceção e desenho do estudo; B.S, A.C implementação experimental; C.F-P análise de dados; C.F-P, A.C, redação, edição e revisão; C.F-P, A.C, figuras e gráficos; C.F-P supervisão e redação final.

\section{Financiamento}

Não aplicável.

\section{Agradecimentos}

Os autores agradecem a todos os participantes e colaboradores envolvidos no estudo, principalmente aos alunos do $3^{\circ}$ ano da Licenciatura em Ciências da Nutrição da Universidade Lusófona de Humanidades e Tecnologias.

\section{Conflito de Interesses}

A.C., C.F.-P., B.S. não têm conflitos de interesse a reportar. 


\section{References/ Referências}

1. Ferreira-Pêgo C, Morales S, Fabregas J, Martín Y, Tavares N. Anthropometric evaluation of children aged between 3 and 9 years from Canary Island. J Biomed Biopharm Res. 2019;16(2):154-64.

2. Ferreira-Pêgo C, Gaipo S, Parece T, Marinho R, Dias T, Viveiros F, et al. Anthropometric evaluation of pre-school and school age children from Azores archipelago, Portugal. J Biomed Biopharm Res. 2019;16(2):165-75.

3. Ferreira M, Faria V, Sousa B, Tavares N. Evaluation of the nutritional status in pre-school and school children, Madeira Island, Portugal. Biomed Biopharm Res. 2019;16(1):8-18.

4. Morales-Suárez-Varela M, Rubio-López N, Ruso C, Llopis-Gonzalez A, Ruiz-Rojo E, Redondo M, et al. Anthropometric status and nutritional intake in children (6-9 years) in Valencia (Spain): The ANIVA study. Int J Environ Res Public Health. 2015;12(12):16081-95.

5. Sousa B. The Anthropometry in Nutritional and Growth Assessment of Children and Adolescents. J Biomed Biopharm Res. 2017;14(2):1-2.

6. Freitas D, Beunen G, Maia J, Claessens A, Thomis M, Marques A, et al. Tracking of fatness during childhood, adolescence and young adulthood: A 7-year follow-up study in Madeira Island, Portugal. Ann Hum Biol. 2012;39(1):59-67.

7. World Medical Association declaration of Helsinki: Ethical principles for medical research involving human subjects. Vol. 310, JAMA - Journal of the American Medical Association. 2013. p. 2191-4

8. Rito A, Breda J, do Carmo I. Guia de Avaliação do Estado Nutricional Infantil e Juvenil [Internet]. Lisboa: INSA.; 2011. Disponível em: ISBN 9789728643614

9. Wijnhoven TM, van Raaij JM, Spinelli A, Starc G, Hassapidou M, Spiroski I, et al. WHO European Childhood Obesity Surveillance Initiative: body mass index and level of overweight among 6-9-year-old children from school year 2007/2008 to school year 2009/2010. BMC Public Health. 2014;14(1):806.

10. Cole TJ, Bellizzi MC, Flegal KM, Dietz WH. Establishing a standard definition for child overweight and obesity worldwide: international survey. BMJ. 2000;320(7244):1240-3

11. Lo K, Wong M, Khalechelvam P, Tam W. Waist-to-height ratio, body mass index and waist circumference for screening paediatric cardio-metabolic risk factors: a meta-analysis. Obes Rev. 2016;17(12):1258-75.

12. Wijnhoven TM, Van Raaij JM, Spinelli A, Starc G, Hassapidou M, Spiroski I, et al. WHO European Childhood Obesity Surveillance Initiative: Body mass index and level of overweight among 6-9-year-old children from school year 2007/2008 to school year 2009/2010. BMC Public Health. 2014;14(1).

13. Rito AI, Paixão E, Carvalho MA, Ramos C. Childhood Obesity Surveillance Initiative: COSI Portugal 2010. 2012;1-37.

14. Freitas AI, Moreira C, Santos AC. Time trends in prevalence and incidence rates of childhood overweight and obesity in Portugal: Generation XXI birth cohort. Int J Obes. 2019;43(2):424-7.

15. Freedman DS, Mei Z, Srinivasan SR, Berenson GS, Dietz WH. Cardiovascular Risk Factors and Excess Adiposity Among Overweight Children and Adolescents: The Bogalusa Heart Study. J Pediatr. 2007;150(1):12-17.e2.

16. Aranceta-Bartrina J, Pérez-Rodrigo C. Determinants of childhood obesity: ANIBES study. Nutr Hosp. 2016;33(Suppl 4):17-20.

17. Nepper MJ, Chai W. Parents' barriers and strategies to promote healthy eating among school-age children. Appetite. 2016;103:157-64. 